\title{
$\beta$-Adrenergic facilitation of synaptic plasticity in the rat basolateral amygdala in vitro is gradually reversed by corticosterone
}

\author{
Zhenwei Pu, ${ }^{1}$ Harm J. Krugers, and Marian Joëls \\ Swammerdam Institute for Life Sciences-Center for NeuroScience, University of Amsterdam, Amsterdam 1098 SM, \\ The Netherlands
}

\begin{abstract}
The rat basolateral amygdala is important for emotional learning; this is modulated by noradrenaline and corticosterone. We report that the $\beta$-adrenergic agonist isoproterenol markedly enhances synaptic plasticity induced in the basolateral amygdala by a weak stimulation paradigm but is ineffective with stronger protocols. Simultaneous application of corticosterone gradually reversed the facilitatory effect of isoproterenol. When corticosterone was briefly applied several hours prior to isoproterenol, facilitatory effects of the $\beta$-agonist were entirely suppressed. This suggests that in the basolateral amygdala, $\beta$-adrenergic influences promote synaptic plasticity; this is gradually normalized by corticosterone, preventing the network from overshooting.
\end{abstract}

The amygdala is crucially involved in the modulation of emotional memory (Cahill and McGaugh 1998; LeDoux 2000; McGaugh 2004; Richter-Levin 2004), as clearly demonstrated in the animal model of fear conditioning (LeDoux et al. 1990; Romanski et al. 1993; Rogan et al. 1997; Nader et al. 2001; Blair et al. 2003). It was also proposed that the amygdala - that is, the basolateral nucleus (BLA) — can modulate memory-related processes in other brain regions, e.g., the hippocampus (McGaugh et al. 1996; Akirav and Richter-Levin 2002; Kim and Diamond 2002; Pare 2003; RichterLevin and Akirav 2003; Roozendaal 2003; Richter-Levin 2004; Roozendaal et al. 2006a); thus, memory traces constructed in the hippocampus that are "emotionally tagged" have a competitive advantage for retention (Richter-Levin and Akirav 2003; Diamond et al. 2005; Vouimba et al. 2007).

Within the BLA, multiple neuromodulatory systems influence memory, including the noradrenergic and glucocorticoid systems (Quirarte et al. 1997; Roozendaal 2003; Roozendaal et al. 2006a). Animal behavioral studies suggested that noradrenergic activity within the BLA plays a central role in mediating a memoryenhancing effect, while glucocortoid receptor (GR) activation exerts a "permissive" function (Roozendaal et al. 2002, 2006b). However, experiments in which the two hormones were not given concurrently showed that glucocorticoids may otherwise suppress the noradrenergic effect (Borrell et al. 1984). This suggests that the interactive hormonal functions affecting the memory systems are not always uniform.

Support for this nonuniformity was recently obtained in the hippocampal DG (Pu et al. 2007). Corticosterone time-dependently modulated noradrenergic action on long-term potentiation (LTP), the best-described neurobiological substrate of learning and memory to date (Goosens and Maren 2002; Martin and Morris 2002; Morris 2003). Thus, $\beta$-adrenergic facilitation of LTP was accelerated if corticosterone was coapplied with the $\beta$-agonist isoproterenol, but suppressed if corticosterone was transiently applied several hours before the $\beta$-agonist (Pu et al. 2007). In view of the behavioral observations that $\beta$-agonists and glucocorticoids both affect memory processes involving the BLA (Roozendaal et al.

'Corresponding author.

E-mail Z.Pu@uva.nl; fax 31-20-5257709.

Article is online at http://www.learnmem.org/cgi/doi/10.1101/lm.1272409.
2002), we here investigated the time-dependent hormonal actions in this region.

Male Wistar rats (200-300 g; Harlan) were group housed, with a 12/12-h light-dark cycle (lights on at 8:00 a.m.). The Animal Committee for Bioethics of University of Amsterdam approved all experiments. Animals were decapitated around $10 \mathrm{a} . \mathrm{m}$. The brain was removed from the skull and immersed in chilled buffer as previously described (Pu et al. 2007); 500- $\mu \mathrm{m}$-thick coronal slices containing the basolateral amygdala were made with a vibroslicer (Leica VT1000S). Slices were maintained in artificial cerebrospinal fluid (aCSF) (see $\mathrm{Pu}$ et al. 2007) for $>1 \mathrm{~h}$ before transfer to the recording chamber $\left(30^{\circ} \mathrm{C}-32^{\circ} \mathrm{C}\right)$, where slices were kept submerged.

The lateral amygdala (LA) supplies one of the major afferent pathways to the BLA (Pitkanen et al. 1995; Wang et al. 2002). A bipolar stimulation electrode $(60 \mu \mathrm{m}$ in diameter, stainless steel, insulated except for the tip) was placed in the LA to evoke field excitatory postsynaptic potentials in the BLA (glass microelectrodes, impedance 2-5 M $\Omega$, filled with aCSF). Stimulus intensity was adjusted to evoke synaptic responses around half-maximal amplitude. The field potential signal in the LA-BLA pathway was characterized by a negative-going waveform (Fig. 1A). Signals followed $100 \mathrm{~Hz}$ of stimulation (Fig. 1B) and were reversibly blocked by the AMPA receptor antagonist CNQX $(10 \mu \mathrm{M})$ (Fig. 1C). Therefore, they represented AMPA-receptor-mediated, monosynaptic responses (Rammes et al. 2000; DeBock et al. 2003; Schimanski and Nguyen 2005).

Three protocols were tested for their ability to induce LTP in the LA-BLA pathway: (1) $5 \times(100 \mathrm{~Hz} \times 1 \mathrm{sec})$, the strongest protocol; (2) $1 \times(100 \mathrm{~Hz} \times 1 \mathrm{sec})$; and (3) weak theta-burst stimulation (TBS). Synaptic potentiation after tetanus was expressed relative to baseline (average of 25 min pre-tetanus). A two-tailed, paired Student's $t$-test was used to compare synaptic responses before versus after high-frequency stimulation within each group. The general linear model for repeated measures (GLM) was performed for between-group comparisons, followed by post hoc least significant difference (LSD) multiple comparison tests. Unless stated otherwise, between-group comparisons were performed for (1) the entire 60-min post-tetanus period and (2) the final part of the post-tetanus period (50-60 min). 
A
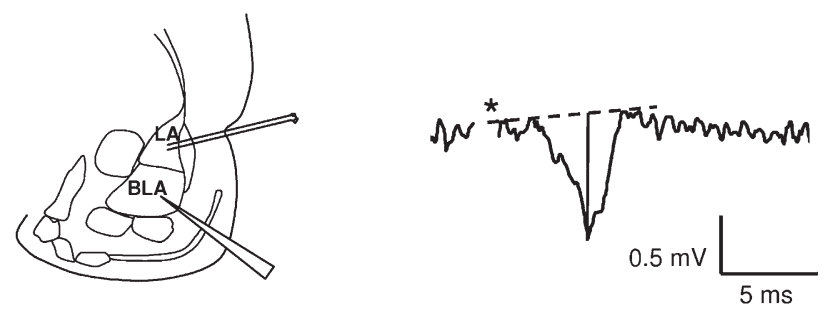

B

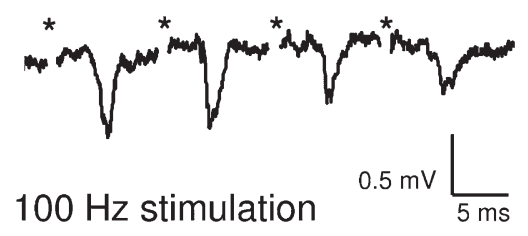

C
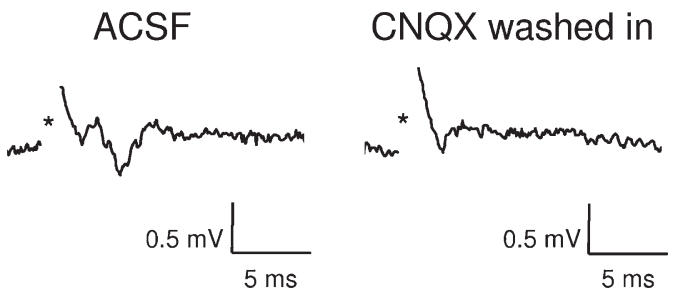

\section{CNQX washed out}

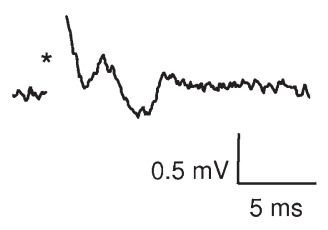

Figure 1. Field potential signals as induced in the LA-BLA pathway that represents a monosynaptic, AMPA-mediated response. $(A)$ Positioning of the stimulating electrode and the recording electrode at their respective sites within the lateral and basolateral amygdala. The magnitude of the recorded field potential signal was measured by projecting the most negative point of the negative-going signal onto a line that connected the onset of the signal and the most positive point during its decay phase. The depicted signal represents an average of the responses to four consecutive stimuli. $(B)$ This signal could follow multiple pulses of stimulation at $100 \mathrm{~Hz}$ without failure. (This trace shows a nonaveraged signal.) (C) The signal was abolished in the presence of the AMPA-receptor antagonist CNQX (10 $\mu \mathrm{M})$, but restored when the drug was cleared from the medium. Signals represent averaged responses to four consecutive stimuli. $\left(^{*}\right)$ Indicates where the stimulus artifact appeared.

LTP was effectively induced by the strongest and intermediate protocols (Fig. 2A,B); over 60 min after tetanus, signals amounted to $152 \% \pm 9 \%$ (mean amplitude \pm SEM, $n=5)$ and $153 \% \pm 10 \%(n=$ 5) of baseline, respectively, indicating significant increases (both $P<$ 0.01). During the final $10 \mathrm{~min}$ of the recording period (50-60 min post-tetanus), strong and significant potentiation was observed in both cases $(156 \% \pm 13 \%, P<0.05$; and $157 \% \pm 14 \%, P<0.05$, respectively). In contrast, TBS did not result in stable LTP (Fig. 2C). The potentiation after tetanus only amounted to $113 \% \pm 9 \%(n=5)$ over $60 \mathrm{~min}$ and $108 \% \pm 10 \%$ over the last $10 \mathrm{~min}$ (both $P>0.05$ ). For these three protocols, over the entire 60-min post-tetanus period, there was an overall difference $\left(F_{(2,12)}=5.79, P<0.05\right)$. Post hoc tests did not indicate any difference between the strongest and intermediate protocols $(P>0.05)$; the effect of TBS, though, was significantly different from the other two (both $P<0.05$; Fig. 2D). This suggests that a single train of $100-\mathrm{Hz}$ pulses (for $1 \mathrm{sec}$ ) was adequate to produce amygdala LTP in our setting. This is partly in accordance with literature showing that LTP is consistently found with five trains of $100 \mathrm{~Hz}$ (1 sec) (Rammes et al. 2000; DeBock et al. 2003; Schimanski and Nguyen 2005), while fewer trains only result in transient potentiation (Rammes et al. 2000).

Noradrenaline is known to bidirectionally affect the BLA. Generally, inhibitory actions occur via $\alpha 2$-adrenergic receptors
(Ferry et al. 1997; DeBock et al. 2003; Buffalari and Grace 2007), whereas $\beta$ adrenergic receptors facilitate excitatory transmission and synaptic potentiation in the BLA (Huang et al. 1996; Ferry et al. 1997; Wang et al. 1999) or LA (Huang et al. 2000). We here tested how $1.0 \mu \mathrm{M}$ of the $\beta$-adrenergic agonist (-)-isoproterenol (+)-bitartrate (Sigma-Aldrich) affects synaptic plasticity with the three protocols. This concentration was sufficient to affect LTP in the DG (Pu et al. 2007); moreover, it is the lowest dose that yields clear effects on single neuron synaptic responses in the BLA (L. Liebmann, $\mathrm{H}$. Karst, and M. Joëls, unpubl.). When isoproterenol was applied for $15 \mathrm{~min}$ before and during the strongest high-frequency stimulation protocol, long-lasting potentiation was apparent $(155 \% \pm 18 \%$ of baseline over $60 \mathrm{~min}, n=4 ; 151 \% \pm 15 \%$ over the last $10 \mathrm{~min}, P<0.05$; Fig. $2 \mathrm{~A}$ ), but there was no difference between the vehicle- and isoproterenol-treated slices (neither over $60 \mathrm{~min}$ nor the last $10 \mathrm{~min}$, both $P>0.05$; Fig. $2 \mathrm{D}$ ). With the intermediate protocol $[1 \times(100 \mathrm{~Hz} \times 1 \mathrm{sec})]$, again, significant potentiation was observed with isoproterenol $(n=5 ; 143 \% \pm$ $8 \%$ of baseline, over $60 \mathrm{~min}$; and $143 \% \pm$ $9 \%$ over the last $10 \mathrm{~min}$, both $P<0.01$; Fig. $2 \mathrm{~B}$ ), not different from the vehicletreated group (both $P>0.05$, over $60 \mathrm{~min}$ and the last $10 \mathrm{~min} ;$ Fig. 2D). In contrast, when TBS was applied, isoproterenol significantly enhanced synaptic potentiation $(n=4 ; 161 \% \pm 10 \%$ of baseline over $60 \mathrm{~min}, P<0.01$; and $166 \% \pm 17 \%$ over the last $10 \mathrm{~min}, P<0.05$; Fig. $2 \mathrm{C}$ ) in comparison with the vehicle condition (both over $60 \mathrm{~min}$ and the final $10 \mathrm{~min}$, $P<0.01$; Fig. 2D). We conclude that modulation of synaptic plasticity in the LA-BLA pathway by a $\beta$-adrenergic agonist is only seen with mild synaptic strengthening, and not when synaptic strength is already maximally boosted.

We next considered the influence of corticosterone (SigmaAldrich, $100 \mathrm{nM}$ ) on LTP. Corticosterone perfusion just before and during TBS (i.e., without isoproterenol) resulted in marginal potentiation over $60 \mathrm{~min}$ after high-frequency stimulation $(114 \% \pm 5 \%, n=5, P<0.05$; Fig. 3B). Over time, potentiation slightly declined so that it was no longer significant during the last $10 \min (111 \% \pm 8 \%, P=0.27 ;$ Fig. 3B,D). Interestingly, application of corticosterone in conjunction with $1 \times(100 \mathrm{~Hz} \times 1 \mathrm{sec})$ also showed a slow and gradual reduction in the level of potentiation compared to vehicle conditions $(P<0.05$ for the final $10 \mathrm{~min}$; Fig. $3 \mathrm{~A})$. This was not seen with the strongest stimulation protocol.

Next, the ability of corticosterone to alter the facilitating effects of isoproterenol on TBS was studied. In the between-group comparison, we were particularly interested in the initial stage of potentiation, since rapid interactive actions of corticosterone and isoproterenol within this time window were earlier described for the $\mathrm{DG}(\mathrm{Pu}$ et al. 2007). For the combined treatment, a significant level of potentiation $(142 \% \pm 11 \%, P=0.01$; Fig. 3B) was observed during the first $10 \mathrm{~min}$ after tetanus. Comparing the coapplication, isoproterenol only, corticosterone only, and the 
A

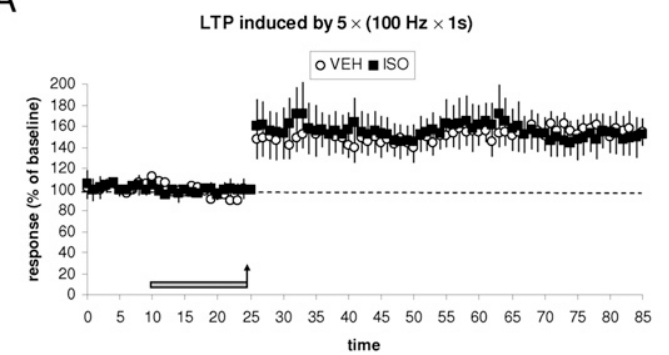

C

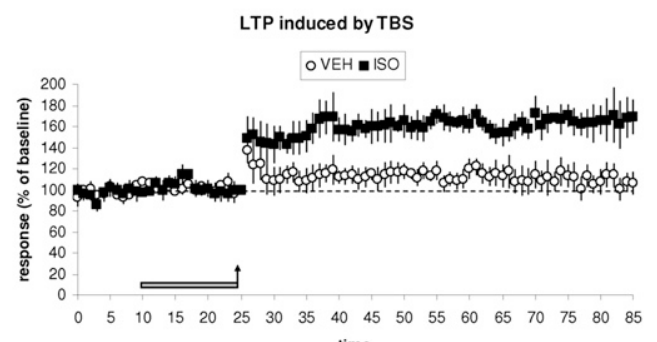

time
B

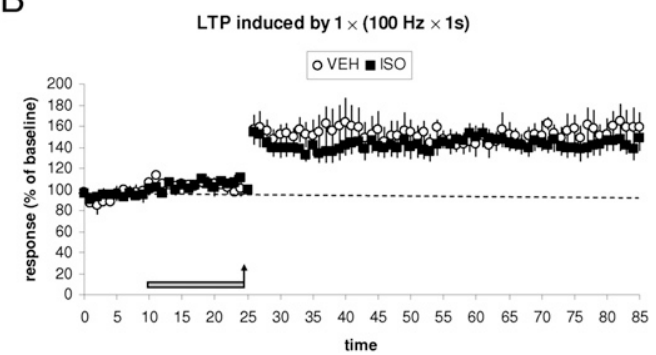

D

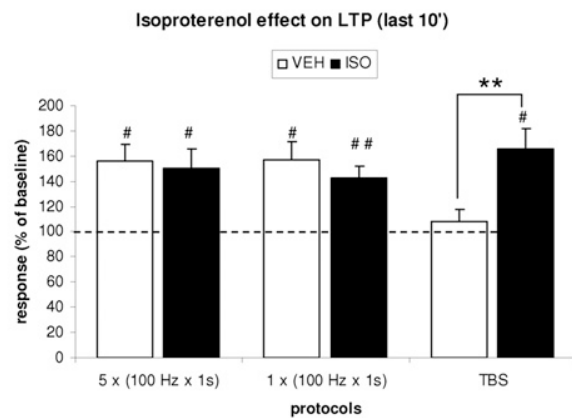

Figure 2. The effect of isoproterenol on synaptic plasticity in the basolateral amygdala with three stimulation protocols of different strengths. ( $A$ ) Strong tetanic stimulation, using five trains of $100 \mathrm{~Hz}$ for $1 \mathrm{sec}$, induced stable LTP in the LA-BLA pathway. Administration of isoproterenol (ISO, $1 \mu \mathrm{M}, n=4)$ instead of vehicle (VEH, $n=5)$ did not affect the degree of potentiation. Horizontal gray bar indicates perfusion with ISO or VEH; the tetanus was given at $t=25 \mathrm{~min}$ (arrow). (B) Similarly, tetanic stimulation with a single train of $100 \mathrm{~Hz}$ for 1 sec yielded stable LTP in the LA-BLA pathway under the VEH condition $(n=5)$. Perfusion with ISO $(n=5)$ resulted in a comparable level of potentiation. (C) With a weak stimulation paradigm (theta-burst stimulation, TBS), only brief post-tetanic potentiation was observed under the VEH condition $(n=5)$. If ISO was perfused just before and during TBS ( $n=4)$, a marked and stable form of synaptic potentiation was achieved. (D) Mean values (+ SEM) representing the averaged responses during the final 10 min of the post-tetanus recording period. Under VEH conditions (white column), both the strong and intermediate stimulation paradigms resulted in stable and significant potentiation in comparison to the pre-tetanus baselines; comparable results were found for ISO perfusion conditions (black column). TBS did not induce significant potentiation under the VEH condition, whereas strong potentiation was observed if ISO was rapidly perfused. (\#) $P<0.05$; (\#\#) $P<0.01$, compared with the individual pre-tetanus baseline. $\left(^{* *}\right) P<0.01$, compared between the groups.

vehicle-treated group, there was an overall difference during the first 10 min after tetanus $\left(F_{(3,17)}=3.53, P<0.05\right)$. Importantly, though, signals recorded after coapplication of corticosterone and isoproterenol did not differ from those seen after isoproterenol application only $(P>0.05)$, unlike the DG (Pu et al. 2007). Notably, in the DG, isoproterenol mediated a gradually developing enhancement of synaptic strength, a process that was accelerated by corticosterone. In the BLA, however, facilitation by isoproterenol is visible immediately after TBS; this leaves little room for further acceleration by corticosterone. Possibly the enhancing effects of corticosterone could be revealed if even lower concentrations of isoproterenol were applied; we refrained from doing so, because lower concentrations of isoproterenol do not give consistent effects in single cell recordings (L. Liebmann, H. Karst, and M. Joëls, unpubl.).

Interestingly, there was an overall difference $\left(F_{(3,17)}=4.09, P<\right.$ 0.05 ) between the four groups during the last $10 \mathrm{~min}$ (50-60 min, post-TBS). Post hoc comparisons demonstrated significant differences between the isoproterenol-treated group on the one hand and the vehicle-treated $(P<0.01)$, corticosterone-treated $(P<0.01)$, or coapplication groups, on the other hand $(P<0.05$; Fig. $3 D)$. The comparison between the groups treated with isoproterenol only versus isoproterenol plus corticosterone is most meaningful. Importantly, these two groups did not differ during the first $30 \mathrm{~min}$ post-TBS $(P=0.140)$, but started to deviate significantly from 50 min post-tetanus onward ( $P=0.032$ during the final $10 \mathrm{~min}$ ). This supports a delayed gene-mediated rather than rapid nongenomic corticosteroid action. Thus, in the BLA, corticosterone did not rapidly facilitate but, rather, slowly diminished the facilitation of synaptic strengthening caused by $\beta$-adrenergic activation.
To further examine the delayed effect by corticosterone, slices were incubated for $20 \mathrm{~min}$ with corticosterone $>2 \mathrm{~h}$ in advance of TBS. Corticosterone preincubation did not significantly alter the absolute field response amplitude compared to $\mathrm{VEH}$ treatment (mean \pm SEM in VEH: $0.59 \pm 0.06 \mathrm{mV}, n=5$; CORT preincubation: $0.66 \pm 0.04 \mathrm{mV}, n=12 ; P=0.33$ ), indicating that hormone treatment by itself did not induce a slow decline in baseline field responses. With preincubation of corticosterone, only a transient post-tetanus potentiation was seen; over $60 \mathrm{~min}$ the synaptic responses amounted to $109 \% \pm 7 \%(n=5, P>0.05$; Fig. 3C), with a declination to $89 \% \pm 9 \%(P>0.05)$ during the final $10 \mathrm{~min}$. If isoproterenol was rapidly perfused to slices that were previously incubated with corticosterone, the isoproterenol-mediated facilitation of post-TBS signals was not seen at all (Fig. 3C); synaptic responses amounted to $118 \% \pm 15 \%(n=6, P>0.05)$ and $105 \% \pm$ $12 \%(P>0.05)$ during the first and the last $10 \mathrm{~min}$ after tetanization, respectively. Comparison during the first $10 \mathrm{~min}$ after tetanus among the four experimental groups revealed no overall difference $\left(F_{(3,16)}=1.43, P>0.05\right)$ and no between-group differences. However, for the last $10 \mathrm{~min}$ (50-60 $\mathrm{min}$, post-TBS), an overall difference was identified $\left(F_{(3,16)}=6.96, P<0.01\right)$. As shown in Figure 3D (50-60 min, post-TBS), post-hoc analysis among all groups revealed a significant difference between corticosterone preincubation prior to isoproterenol perfusion and isoproterenol perfusion only $(P<0.01)$.

We conclude that a delayed suppressive effect of corticosterone on $\beta$-adrenergic facilitation of synaptic plasticity was evident from our study. This was not driven by any drug-mediated changes in baseline transmission as there was no apparent difference with 
A

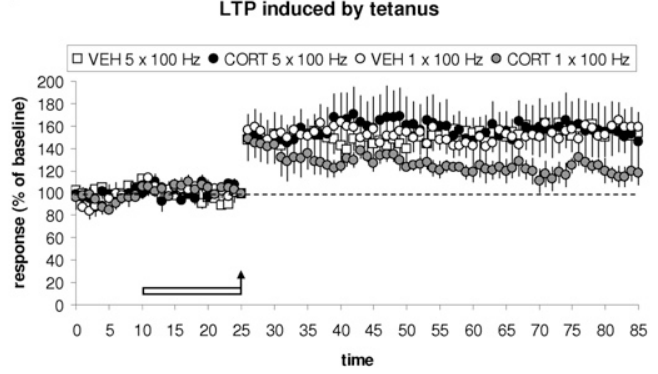

C

LTP induced by TBS

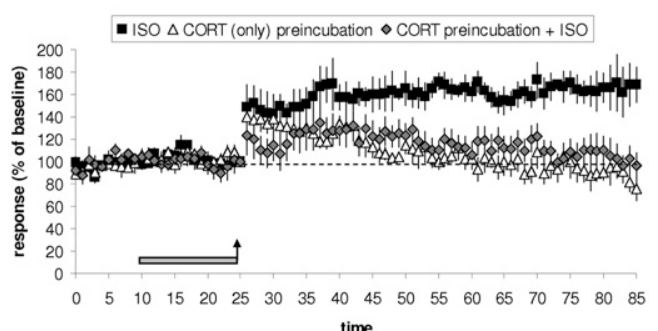

B

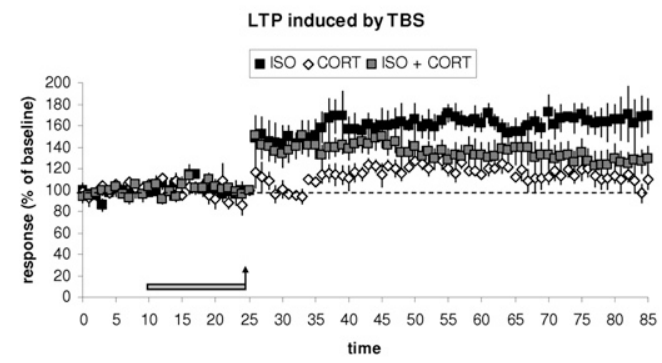

D
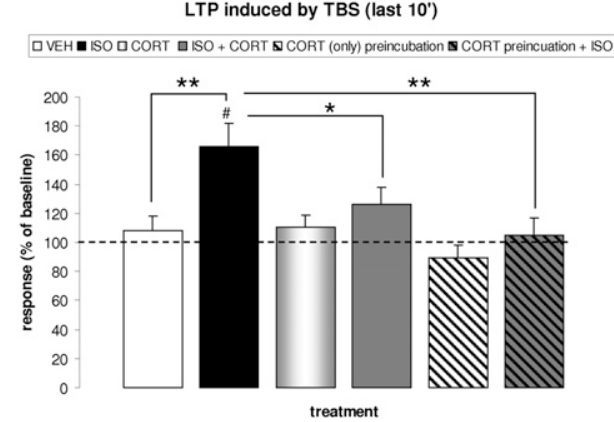

Figure 3. Modulation by isoproterenol and/or corticosterone of synaptic plasticity in the LA-BLA pathway with theta-burst stimulation. (A) Application of corticosterone (100 nM, indicated by the horizontal white bar) just prior to and during high-frequency stimulation gradually reversed synaptic strengthening seen with an intermediate strength stimulation protocol [CORT, $1 \times(100 \mathrm{~Hz} \times 1 \mathrm{sec}) ; n=6$ ] but was ineffective with maximal synaptic strengthening using [CORT, $5 \times(100 \mathrm{~Hz} \times 1 \mathrm{sec}) ; n=5]$. Thus, after corticosterone treatment, the averaged signal $50-60 \mathrm{~min}$ after $1 \times(100 \mathrm{~Hz} \times 1 \mathrm{sec})$ stimulation was reduced significantly $(P<0.05)$ compared to that seen in the matching vehicle groups $(0.009 \%$ ethanol). (B) Brief perfusion with ISO led to marked LTP after TBS $(n=4)$. The horizontal bar indicates the perfusion period; the arrow points to the moment of tetanus. When 100 nM corticosterone was perfused together with isoproterenol (ISO + CORT, $n=7$ ), a significant level of LTP was seen over the entire 60 min post-TBS period; however, it declined during the late stage of potentiation. Perfusion of corticosterone alone (CORT, $n=5$, diamonds) before and during TBS induced weak synaptic potentiation $(114 \% \pm 5 \%$ ) as determined over the entire 60 -min recording period post-TBS, although this was no longer significant over the final $10 \mathrm{~min}(111 \% \pm 8 \%)$; the latter is similar to the lack of effect of VEH (this is depicted in Fig. 2, not shown in this graph). It should be noted that the signal in the CORT group was slightly variable after TBS. Shortly after TBS it declined to baseline. Follow-up analysis revealed that this can be explained by chance fluctuations in the signal (which occurred at the same frequency during baseline as after TBS and which in other experimental groups resulted in variability at other time points). After the brief decline between $t=29$ and $33 \mathrm{~min}$, the signal developed into a marginal form of potentiation over 60 min. For instance, between $t=50$ and $65 \mathrm{~min}$, field responses were significantly enhanced compared to baseline $(P<0.05)$. Importantly, though, the degree of potentiation between $t=50$ and $65 \mathrm{~min}$ was very small compared to that seen in the ISO group over the same period of time $(P=0.01)$ and not significantly different from the signal in the VEH-treated group $(P=0.617)$. (C) If corticosterone was briefly $(20 \mathrm{~min})$ administered more than $2 \mathrm{~h}$ in advance of isoproterenol and TBS (CORT preincubation + ISO, $n=6$ ), any form of LTP attributable to isoproterenol-mediated facilitation was completely lost. Pretreatment with corticosterone alone (CORT only preincubation, $n=5$ ) did not result in significant synaptic potentiation, similar to VEH treatment (not shown in this graph). (D) Between-group comparisons (mean + SEM) show that isoproterenol alone enhanced synaptic responses after TBS (50-60 min after TBS). This facilitatory effect of $\beta$-adrenergic activation was not further enhanced by concomitant application of corticosterone (through its rapid effect); in contrast, a decrease was noticed during the last $10 \mathrm{~min}$ of the post-tetanus period. The facilitative effect of $\beta$-adrenergic activation was completely prevented if corticosterone was applied more than $2 \mathrm{~h}$ in advance (through its delayed effect). (VEH: white column; ISO: black column; CORT: gradiently gray column; ISO + CORT: full gray column; CORT only preincubation: striped white column; CORT preincubation + ISO, striped gray column.) (\#) $P<0.05$, compared with the individual pre-tetanus baseline; $\left(^{*}\right) P<0.05 ;\left({ }^{* *}\right) P<0.01$, compared between the groups.

regard to the baseline transmission during perfusion of different hormone(s) (pooled data, including all three stimulation protocols: $\left.F_{(4,69)}=0.16, P>0.05\right)$ nor between-group differences for any individual comparison against the vehicle treatment (all $P>0.05$ ). This differs from earlier studies reporting that $15 \mu \mathrm{M}$ isoproterenol increases AMPA-receptor-mediated responses via presynaptic enhancement of $\mathrm{P} / \mathrm{Q}$ calcium currents (Huang et al. 1996), while postsynaptically isoproterenol enhances NMDA-receptor-mediated currents (Huang et al. 1998a). The lack of effect in the present study is probably due to the much lower concentration of the $\beta$-agonist; thus, we cannot exclude that moderate as opposed to high concentrations of the $\beta$-agonist employ different mechanisms, for example, on presynaptic processes. The fact that corticosterone was ineffective is in line with a recent study reporting no effects of $(100 \mathrm{nM})$ corticosterone on excitatory postsynaptic potentials evoked in identified BLA principal neurons (Duvarci and Pare 2007).
Overall, the present electrophysiological studies indicate a facilitating role of noradrenaline via $\beta$-adrenergic receptors on synaptic plasticity in the BLA, which is in line with behavioral observations pinpointing a quintessential function of BLA $\beta$ receptors in consolidation of emotionally arousing events (Ferry et al. 1999; Roozendaal et al. 2006a). Our findings suggest that activation of $\beta$-adrenergic receptors is particularly effective in relatively weak learning paradigms, but less so with stronger forms of aversive learning. Under the present experimental conditions, we did not find evidence for a permissive role of corticosterone in the BLA. This seemingly contrasts with behavioral observations (Roozendaal et al. 2002, 2006a). However, it should be realized that our experiments were carried out in an isolated preparation, whereas the BLA in vivo functions in a network of interconnected brain regions. Moreover, at this stage we cannot exclude the possibility that corticosterone may accelerate 
noradrenergic function with a lower degree of $\beta$-adrenoceptor activation.

While corticosterone did not promote the action of isoproterenol, a consistent delayed suppressive effect was observed. The delayed onset supports a role of nuclear receptors. Of these, the glucocorticoid receptor is most likely involved. Thus, a pulse of $100 \mathrm{nM}$ corticosterone is sufficient to activate these receptors in vitro (Karst et al. 2000). Importantly, we prepared slices from adrenally intact animals, in which most of the nuclear mineralocorticoid receptors are already activated, so that a pulse of corticosterone will predominantly occupy glucocorticoid receptors. Interestingly, corticosterone by itself already seemed to slowly suppress synaptic potentiation-at least with low to intermediate synaptic strengthening - which could signify that a late phase of synaptic potentiation was specifically targeted. In the LA and the DG, this late phase of LTP was found to depend on protein kinase A and mitogen-activated protein kinase (Huang et al. 2000; $\mathrm{Wu}$ et al. 2006), second messengers that are also influenced by $\beta$-agonists (Huang et al. 1998a,b; Price et al. 2004). By sharing a common endpoint, acting in an opposite direction, corticosterone could gradually reduce the efficacy of noradrenergic effects via the $\beta$-adrenergic receptor. However, this awaits further study.

The gradual suppression of $\beta$-adrenergic signaling by corticosterone fits well with results at the behavioral level, showing that post-learning administration of adrenaline to adrenalectomized rats facilitated memory retention in a passive avoidance paradigm, a facilitation that was largely impaired by pretreatment with corticosterone (Borrell et al. 1984). Interestingly, we did not only see a delayed suppressive effect when corticosterone was administered several hours in advance of isoproterenol, but also when the two compounds were given simultaneously. As intraBLA elevations in the levels of noradrenaline and corticosterone are, indeed, expected to occur within a restricted time window after stress exposure, this strongly argues that noradrenaline, via $\beta$-adrenoreceptors, rapidly promotes activity-dependent synaptic plasticity in the BLA, which is subsequently and slowly normalized by corticosterone. If so, corticosteroids would serve to contain the initial stress response and prevent it from inappropriate overshooting.

\section{Acknowledgments}

We thank Professor M. Witter for discussions on slice preparation development. Z.P. was supported by grant no. 912-04-042 from the Dutch Organization for Scientific Research NWO.

\section{References}

Akirav, I. and Richter-Levin, G. 2002. Mechanisms of amygdala modulation of hippocampal plasticity. J. Neurosci. 22: 9912-9921.

Blair, H.T., Tinkelman, A., Moita, M.A., and LeDoux, J.E. 2003. Associative plasticity in neurons of the lateral amygdala during auditory fear conditioning. Ann. N.Y. Acad. Sci. 985: 485-487.

Borrell, J., de Kloet, E.R., and Bohus, B. 1984. Corticosterone decreases the efficacy of adrenaline to affect passive avoidance retention of adrenalectomized rats. Life Sci. 34: 99-104.

Buffalari, D.M. and Grace, A.A. 2007. Noradrenergic modulation of basolateral amygdala neuronal activity: Opposing influences of $\alpha-2$ and $\beta$ receptor activation. J. Neurosci. 27: 12358-12366.

Cahill, L. and McGaugh, J.L. 1998. Mechanisms of emotional arousal and lasting declarative memory. Trends Neurosci. 21: 294-299.

DeBock, F., Kurz, J., Azad, S.C., Parsons, C.G., Hapfelmeier, G., Zieglgansberger, W., and Rammes, G. 2003. $\alpha_{2}$-Adrenoreceptor activation inhibits LTP and LTD in the basolateral amygdala: Involvement of $\mathrm{G}_{\mathrm{i} / \mathrm{o}}$-protein-mediated modulation of $\mathrm{Ca}^{2+}$-channels and inwardly rectifying $\mathrm{K}^{+}$-channels in LTD. Eur. J. Neurosci. 17: 1411-1424.

Diamond, D.M., Park, C.R., Campbell, A.M., and Woodson, J.C. 2005. Competitive interactions between endogenous LTD and LTP in the hippocampus underlie the storage of emotional memories and stressinduced amnesia. Hippocampus 15: 1006-1025.
Duvarci, S. and Pare, D. 2007. Glucocorticoids enhance the excitability of principal basolateral amygdala neurons. J. Neurosci. 27: 4482-4491.

Ferry, B., Magistretti, P.J., and Pralong, E. 1997. Noradrenaline modulates glutamate-mediated neurotransmission in the rat basolateral amygdala in vitro. Eur. J. Neurosci. 9: 1356-1364.

Ferry, B., Roozendaal, B., and McGaugh, J.L. 1999. Basolateral amygdala noradrenergic influences on memory storage are mediated by an interaction between $\beta$ - and $\alpha 1$-adrenoceptors. J. Neurosci. 19: 5119-5123.

Goosens, K.A. and Maren, S. 2002. Long-term potentiation as a substrate for memory: Evidence from studies of amygdaloid plasticity and Pavlovian fear conditioning. Hippocampus 12: 592-599.

Huang, C.C., Hsu, K.S., and Gean, P.W. 1996. Isoproterenol potentiates synaptic transmission primarily by enhancing presynaptic calcium influx via P- and/or Q-type calcium channels in the rat amygdala. J. Neurosci. 16: 1026-1033.

Huang, C.C., Lin, C.H., and Gean, P.W. 1998a. Potentiation of $N$-methylD-aspartate currents by isoproterenol in the acutely dissociated rat amygdalar neurons. Neurosci. Lett. 253: 9-12.

Huang, C.C., Wang, S.J., and Gean, P.W. 1998b. Selective enhancement of P-type calcium currents by isoproterenol in the rat amygdala. J. Neurosci. 18: $2276-2282$.

Huang, Y.Y., Martin, K.C., and Kandel, E.R. 2000. Both protein kinase A and mitogen-activated protein kinase are required in the amygdala for the macromolecular synthesis-dependent late phase of long-term potentiation. J. Neurosci. 20: 6317-6325.

Karst, H., Karten, Y.J., Reichardt, H.M., de Kloet, E.R., Schütz, G., and Joëls, M. 2000. Corticosteroid actions in hippocampus require DNA binding of glucocorticoid receptor homodimers. Nat. Neurosci. 3: 977-978.

Kim, J.J. and Diamond, D.M. 2002. The stressed hippocampus, synaptic plasticity and lost memories. Nat. Rev. Neurosci. 3: 453-462.

LeDoux, J.E. 2000. Emotion circuits in the brain. Annu. Rev. Neurosci. 23: 155-184.

LeDoux, J.E., Cicchetti, P., Xagoraris, A., and Romanski, L.M. 1990. The lateral amygdaloid nucleus: Sensory interface of the amygdala in fear conditioning. J. Neurosci. 10: 1062-1069.

Martin, S.J. and Morris, R.G. 2002. New life in an old idea: The synaptic plasticity and memory hypothesis revisited. Hippocampus 12: 609-636.

McGaugh, J.L. 2004. The amygdala modulates the consolidation of memories of emotionally arousing experiences. Annu. Rev. Neurosci. 27: 1-28.

McGaugh, J.L., Cahill, L., and Roozendaal, B. 1996. Involvement of the amygdala in memory storage: Interaction with other brain systems. Proc. Natl. Acad. Sci. 93: 13508-13514.

Morris, R.G. 2003. Long-term potentiation and memory. Philos. Trans. $R$ Soc. Lond. B Biol. Sci. 358: 643-647.

Nader, K., Majidishad, P., Amorapanth, P., and LeDoux, J.E. 2001. Damage to the lateral and central, but not other, amygdaloid nuclei prevents the acquisition of auditory fear conditioning. Learn. Mem. 8: 156-163.

Olsson, A. and Phelps, E.A. 2007. Social learning of fear. Nat. Neurosci. 10: 1095-1102.

Pare, D. 2003. Role of the basolateral amygdala in memory consolidation. Prog. Neurobiol. 70: 409-420.

Pitkanen, A., Stefanacci, L., Farb, C.R., Go, G.G., LeDoux, J.E., and Amaral, D.G. 1995. Intrinsic connections of the rat amygdaloid complex: Projections originating in the lateral nucleus. J. Comp. Neurol. 356: 288-310.

Price, D.M., Chik, C.L., and Ho, A.K. 2004. Norepinephrine induction of mitogen-activated protein kinase phosphatase- 1 expression in rat pinealocytes: Distinct roles of $\alpha$ - and $\beta$-adrenergic receptors. Endocrinology 145: 5723-5733.

$\mathrm{Pu}, \mathrm{Z}$., Krugers, H.J., and Joels, M. 2007. Corticosterone time-dependently modulates $\beta$-adrenergic effects on long-term potentiation in the hippocampal dentate gyrus. Learn. Mem. 14: 359-367.

Quirarte, G.L., Roozendaal, B., and McGaugh, J.L. 1997. Glucocorticoid enhancement of memory storage involves noradrenergic activation in the basolateral amygdala. Proc. Natl. Acad. Sci. 94: 14048-14053.

Rammes, G., Steckler, T., Kresse, A., Schutz, G., Zieglgansberger, W., and Lutz, B. 2000. Synaptic plasticity in the basolateral amygdala in transgenic mice expressing dominant-negative cAMP response element-binding protein (CREB) in forebrain. Eur. J. Neurosci. 12: 2534-2546.

Richter-Levin, G. 2004. The amygdala, the hippocampus, and emotional modulation of memory. Neuroscientist 10: 31-39.

Richter-Levin, G. and Akirav, I. 2003. Emotional tagging of memory formation in the search for neural mechanisms. Brain Res. Brain Res. Rev. 43: $247-256$

Rogan, M.T., Staubli, U.V., and LeDoux, J.E. 1997. Fear conditioning induces associative long-term potentiation in the amygdala. Nature 390: 604-607.

Romanski, L.M., Clugnet, M.C., Bordi, F., and LeDoux, J.E. 1993. Somatosensory and auditory convergence in the lateral nucleus of the amygdala. Behav. Neurosci. 107: 444-450. 
Roozendaal, B. 2003. Systems mediating acute glucocorticoid effects on memory consolidation and retrieval. Prog. Neuropsychopharmacol. Biol. Psychiatry 27: 1213-1223.

Roozendaal, B., Quirarte, G.L., and McGaugh, J.L. 2002. Glucocorticoids interact with the basolateral amygdala $\beta$-adrenoceptor-cAMP/cAMP/ PKA system in influencing memory consolidation. Eur. J. Neurosci. 15: 553-560.

Roozendaal, B., Okuda, S., de Quervain, D.J., and McGaugh, J.L. $2006 a$. Glucocorticoids interact with emotion-induced noradrenergic activation in influencing different memory functions. Neuroscience 138: 901-910.

Roozendaal, B., Okuda, S., Van der Zee, E.A., and McGaugh, J.L. 2006b. Glucocorticoid enhancement of memory requires arousal-induced noradrenergic activation in the basolateral amygdala. Proc. Natl. Acad. Sci. 103: 6741-6746.

Schimanski, L.A. and Nguyen, P.V. 2005. Mouse models of impaired fear memory exhibit deficits in amygdalar LTP. Hippocampus 15: 502-517.
Vouimba, R.M., Yaniv, D., and Richter-Levin, G. 2007. Glucocorticoid receptors and $\beta$-adrenoceptors in basolateral amygdala modulate synaptic plasticity in hippocampal dentate gyrus, but not in area CA1. Neuropharmacology 52: 244-252.

Wang, S.J., Cheng, L.L., and Gean, P.W. 1999. Cross-modulation of synaptic plasticity by $\beta$-adrenergic and 5-HT1A receptors in the rat basolateral amygdala. J. Neurosci. 19: 570-577.

Wang, C., Kang-Park, M.H., Wilson, W.A., and Moore, S.D. 2002. Properties of the pathways from the lateral amygdal nucleus to basolateral nucleus and amygdalostriatal transition area. J. Neurophysiol. 87: 2593-2601.

Wu, J., Rowan, M.J., and Anwyl, R. 2006. Long-term potentiation is mediated by multiple kinase cascades involving CaMKII or either PKA or p42/44 MAPK in the adult rat dentate gyrus in vitro. J. Neurophysiol. 95: 3519-3527.

Received October 31, 2008; accepted in revised form November 13, 2008. 


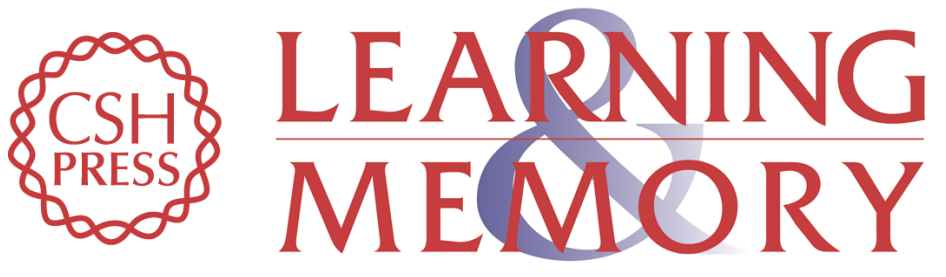

\section{$\beta$-Adrenergic facilitation of synaptic plasticity in the rat basolateral amygdala in vitro is gradually reversed by corticosterone}

Zhenwei Pu, Harm J. Krugers and Marian Joëls

Learn. Mem. 2009, 16:

Access the most recent version at doi:10.1101//m.1272409

References This article cites 44 articles, 14 of which can be accessed free at:

http://learnmem.cshlp.org/content/16/2/155.full.html\#ref-list-1

License

Email Alerting Receive free email alerts when new articles cite this article - sign up in the box at the Service top right corner of the article or click here. 